\title{
Monophasic CIDP Associated with Autoimmune Hemolytic Anemia
}

\author{
S.J. Netherton, C.J. Owen, D.W. Zochodne
}

Can J Neurol Sci. 2014; 41: 290-292

Unlike other autoimmune polyneuropathies, the linkage between chronic inflammatory demyelinating polyneuropathy (CIDP) and other immune mediated disorders is weak. We report a patient who developed monophasic and parallel courses of autoimmune hemolytic anemia (AIHA) and chronic demyelinating polyneuropathy. Both resolved in tandem with corticosteroid therapy.

Distinct from Guillain-Barre Syndrome (GBS) or myasthenia gravis, CIDP has a relatively weak association with other autoimmune disorders. Two previous reports have described patients with Evans syndrome (concurrent AIHA and thrombocytopenia [ITP]) who subsequently developed demyelinating polyneuropathy. In the first report ${ }^{1}$, the patient presented with Evans syndrome and developed GBS several years later; while the second report described a patient with treated Evans syndrome who developed a relapse accompanied by the development of $\mathrm{GBS}^{2}$. Knecht and colleagues ${ }^{3}$ also described a patient with pre-existing CIDP who developed Evans syndrome after a 17 month course of immunomodulatory therapy. Marsh and colleagues ${ }^{4}$ reported a patient with AIHA developing 18 months after treatment of CIDP with alemtuzumab. These rare cases suggest a potential linkage between AIHA (or other autoimmune hematologic disorders) and CIDP. Here, we describe a patient who developed concurrent monophasic and parallel courses of AIHA and CIDP, both of which responded to a short course of corticosteroid therapy.

\section{Case Report}

A previously healthy 85 -year-old right handed man presented with a one week history of increasing shortness of breath with hypoxia in addition to three weeks of generalized, progressive fatigue and weakness. He lost his ability to walk independently. He was diagnosed with left lower lobe community acquired pneumonia, treated with ceftriaxone and clindamycin, but was noted to have a new unexplained anemia. He also reported positive sensory symptoms and stocking sensory loss in his feet for approximately twelve months. At presentation, the patient was anemic with a hemoblogin of $102 \mathrm{~g} / \mathrm{L}$, and a MCV of $87 \mathrm{fL}$. Six months prior to presentation, he had a normal hemoglobin of $155 \mathrm{~g} / \mathrm{L}$. He developed a rapidly progressive decline in his hemoglobin with a nadir of $63 \mathrm{~g} / \mathrm{L}$ by Day 3 with a haptoglobin of $0.02 \mathrm{~g} / \mathrm{L}$ (normal $0.30-2.00 \mathrm{~g} / \mathrm{L}$ ), LDH $263 \mathrm{U} / \mathrm{L}$ (normal 100 - $235 \mathrm{U} / \mathrm{L}$ ) and reticulocytosis of $5.3 \%$ (normal $0.2-2.0 \%$ ) with a positive monospecific direct antiglobulin test (DAT) for IgG (negative for C3). His white blood cell count on presentation was elevated at $18.6 \times 10^{9} / \mathrm{L}$ and the following studies were normal: platelets, electrolytes and renal function, random glucose, and ferritin. Blood and urine cultures were negative, as was a fecal occult blood test conducted on Day 3. The peripheral blood film was reported as showing normochromic normocytic anemia with polychromasia, but no schistocytes or spherocytes were observed. Despite the lack of spherocytes, a diagnosis of warm AIHA was made and treatment with corticosteroids was instituted. Although the use of ceftriaxone was among the risk factors for this disorder, he had a low hemoglobin before the antibiotic was administered and the DAT was positive without the addition of the antibiotic to the test reagents.

While in hospital, his neurological examination noted distal weakness without wasting (finger extensors $4 / 5$; interossei and thumb abduction $4 / 5$, toe dorsiflexion and eversion $4+/ 5$ ), stocking (ankles) and glove (wrists) loss of sensation to light touch and pinprick, loss of vibratory perception, areflexia and gait ataxia. No cranial neuropathies were noted. Electrophysiological studies identified prominent features of primary demyelination: prolonged distal motor latencies, diffuse slowing of conduction velocity, conduction block, and prolonged $F$ wave latencies (Table; Figure). Upper limb sensory potentials were reduced in amplitude with slowing of conduction velocity. The radial sensory nerve action potential was absent. In addition however there was loss of the distal peroneal and tibial compound muscle action potentials and absent lower limb sensory potentials. Despite the presence of weakness, on needle electromyography, there was no abnormal spontaneous activity and motor unit potential configuration was largely normal. Additional investigations for the neuropathy including $\mathrm{B}_{12}$ and folate levels, serum and cerebrospinal fluid (CSF) glucose, toxin and ethanol screens, and a lumbar puncture, revealed only mildly elevated CSF protein $(0.53 \mathrm{~g} / \mathrm{L}$; RR $0.15-0.45 \mathrm{~g} / \mathrm{L})$, but no other abnormalities. Although his disorder was monophasic with a subacute deterioration in motor function, given the chronicity of his sensory symptoms, a diagnosis of probable $\mathrm{CIDP}^{5}$ was made.

From the Departments of Medicine, Division of Hematology \& Hematological Malignancies (SJN, CJO) and Clinical Neurosciences, Division of Neurology (DWZ), Faculty of Medicine, University of Calgary, Calgary, Alberta, Canada.

Received August 19, 2013. Final Revisions Submitted October 8, 2013. Correspondence to: D.W. Zochodne, 168 Heritage Medical Research Bldg, 3330 Hospital Dr. NW, Calgary, Alberta, T2N 4N1, Canada. Email: dzochodn@ucalgary.ca. 


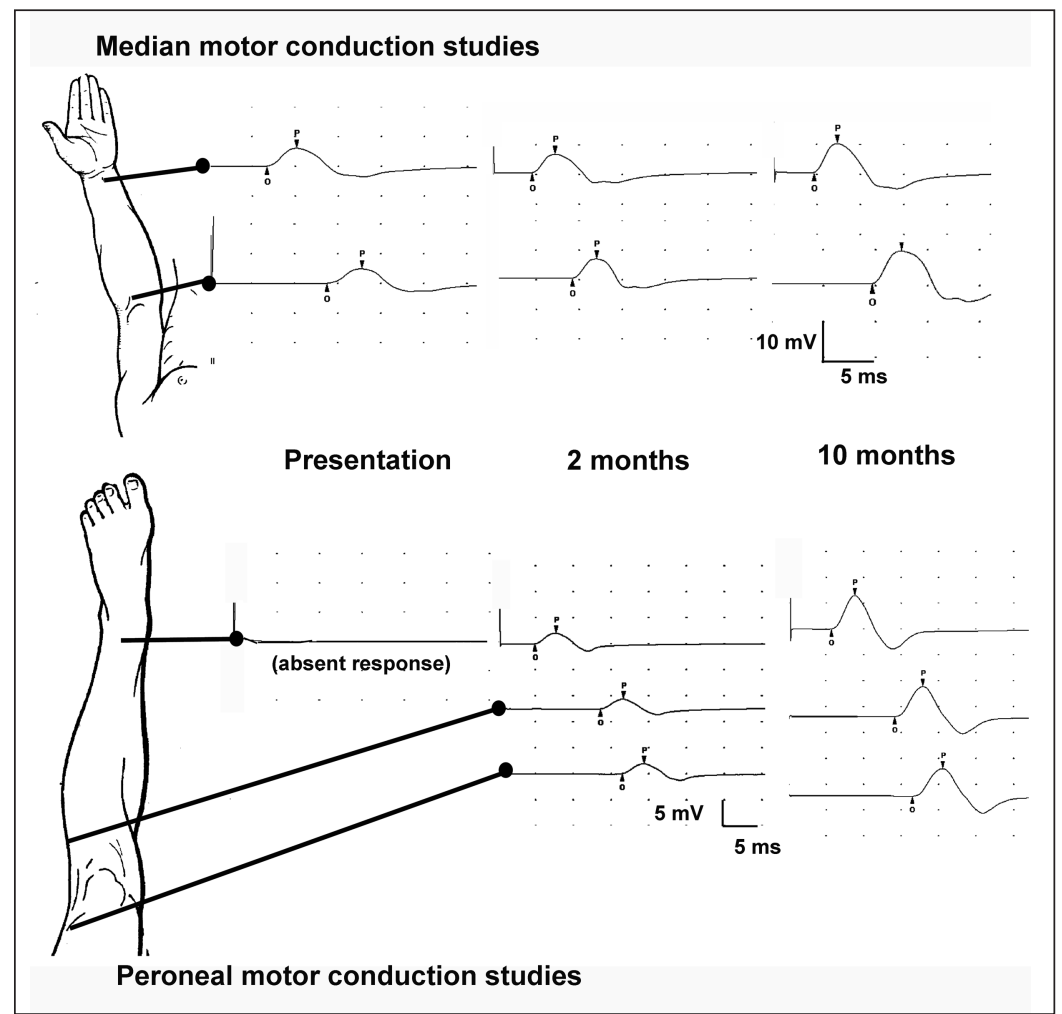

Figure: Illustration of serial compound muscle action potentials (CMAPs) recorded from two (median and peroneal) motor territories in the patient described in this report. In the median nerve, stimulation was carried out at the wrist (distal) and elbow (proximal) with CMAPs recorded from the abductor pollicus brevis muscle from each site of stimulation illustrated over the course of his demyelinating polyneuropathy. In the peroneal nerve, CMAPs were recorded over extensor digitorum brevis with stimulation at the ankle, fibular head and knee as illustrated. Note the improvement in the CMAP amplitudes over time and the progressive shortening in their latency of onset.

Table: Serial electrophysiological results

\begin{tabular}{c|l|l|l|l}
\hline & Date & DML & CV & CMAP (D,P)/SNAP \\
\hline Median motor & Presentation & 6.7 & 25 & $6.0,4.9$ \\
\hline & 2 months & 4.5 & 48 & $6.2,6.5$ \\
\hline Normal & 10 months & 4.7 & 46 & $9.6,9.1$ \\
\hline Median sensory & & $<4.5 \mathrm{~ms}$ & $>50 \mathrm{~m} / \mathrm{s}$ & $>5.0 \mathrm{mV}$ \\
\hline & Presentation & & 33 & 6.1 \\
\hline Normal & 2 months & & 40 & 7.4 \\
\hline Ulnar motor & 10 months & & 37 & 9.9 \\
\hline & & & $>50 \mathrm{~m} / \mathrm{s}$ & $>10 \mathrm{uV}$ \\
\hline Normal & Presentation & 5.8 & 31 & $4.8,3.3,2.9$ \\
\hline Ulnar sensory & 2 months & 3.4 & 48 & $5.8,4.1,3.2$ \\
\hline & 10 months & 3.8 & 51 & $7.2,5.7,5.5$ \\
\hline & & $<4.5 \mathrm{~ms}$ & $>50 \mathrm{~m} / \mathrm{s}$ & $>5.0 \mathrm{mV}$ \\
\hline Normal & Presentation & & 29 & 4.7 \\
\hline Peroneal motor & 2 months & & 45 & 2.4 \\
\hline & 10 months & & 40 & 3.3 \\
\hline & & & $>50 \mathrm{~m} / \mathrm{s}$ & $>10 \mathrm{uV}$ \\
\hline Normal & Presentation & $\mathrm{NR}$ & & \\
\hline Sural sensory & 2 months & 4.9 & 38 & $2.0,1.9,1.9$ \\
\hline & 10 months & 5.7 & 40 & $6.4,5.8,5.3$ \\
\hline Normal & & $<6.2 \mathrm{~ms}$ & $>39 \mathrm{~m} / \mathrm{s}$ & $>2.0 \mathrm{mV}$ \\
\hline & Presentation & $\mathrm{NR}$ & & 2.4 \\
\hline & 2 months & & 40 & 5.9 \\
\hline & 10 months & & $>39 \mathrm{~m} / \mathrm{s}$ & $>6.0 \mathrm{uV}$ \\
\hline
\end{tabular}

$\mathrm{DML}=$ distal motor latency; $\mathrm{CV}=$ conduction velocity; $\mathrm{CMAP}=$ amplitude of compound muscle action potential (mV; distal, proximal); SNAP=amplitude of sensory nerve action potential $(\mathrm{uV})$; Note that ulnar and peroneal motor CMAPs were recorded from stimulation at three sites (ulnar-wrist, below and above elbow; peroneal-ankle, fibular head, knee). 
Following initiation of $70 \mathrm{mg}$ daily prednisone, the patient's hemoglobin normalized over the course of a week, reaching 145 $\mathrm{g} / \mathrm{L}$, with normalization of LDH (147 U/L) and haptoglobin $(1.45 \mathrm{~g} / \mathrm{L})$ three months later. The patient's AIHA was treated with an extended course of prednisone, which was tapered over six months. Neurological recovery also ensued, albeit more slowly with improvements in motor function and electrophysiology. By ten months following his admission, he had only borderline hand weakness and distal sensory loss with dramatic recovery of his electrophysiological abnormalities (Table; Figure). His ability to handle a rifle (professional skeet shooter) had recovered to $80 \%$.

\section{Discussion}

An association between AIHA and CIDP has not previously been reported. None of the other features of the patient's neurological presentation could be attributed to systemic disease or to medications. Also, the chronicity of the demyelinating disorder and its apparent response to prednisone rendered a diagnosis of CIDP rather than GBS. While ceftriaxone can trigger AIHA, a number of the features described above were not compatible with that explanation for his hemolysis. Second and third generation cephalosporins, especially cefotetan and ceftriaxone respectively, can cause AIHA but the exact mechanism by which this occurs is still unknown. The drug may alter antigens on erythrocyte membranes, leading to autoantibody production and cell lysis or new epitopes may be generated by the drug associating with membrane structures causing complement activation and subsequent intravascular cell lysis ${ }^{6}$. Drug-induced hemolysis antibodies are most frequently reported as IgM-type, termed "immune complex type", detectable by positivity for $\mathrm{C} 3$ on the monospecific DAT whereas IgG type antibodies, as found in this case, are uncommon. It is typical to require the addition of the antibiotic to the laboratory reagents to detect a positive DAT in druginduced hemolysis, a feature that was not necessary in this case; though drug or drug-immune complexes in the plasma sample may be adequate to result in a positive DAT in rare cases. In some patients, there is a clear trigger for AIHA; but in many, the mechanism remains unknown. A current theory is that B-cell clones may be altered or de-repressed leading to overproduction of IgG antibodies against autoantigens, or that there may be defective control of IgG autoreactivity by autologous $\operatorname{IgM}^{7}$.

It is not uncommon for patients to manifest multiple autoimmune diseases over the course of their lifetime; however the new, relatively simultaneous onset of multiple autoimmune diseases is quite rare. Our patient developed AIHA in the setting of a rapid deterioration in motor function linked with chronic symptoms of neuropathy, diagnosed as CIDP. Both conditions underwent fulminant exacerbations concurrently. CIDP is suspected to have an immunological basis, but specific provoking antigens have not been discovered ${ }^{5}$. There is no clear link described in the literature between potential epitopes on erythrocytes and myelin; however the nature of the autoimmune disease exacerbation in this patient suggests a single underlying etiology.

\section{REFERENCES}

1. Ala FA, Shearman DJ. A case of autoimmune haemolytic anemia, thrombocytopenia and Landry-Guillain-Barré syndrome. Acta Haematol. 1965;34(6):361-9.

2. Guar S, Sudhathi R, Baumann MA. Acute inflammatory demyelinating polyneuropathy concurrent with Evans syndrome. Southern Med J. 2003;96(1):106-7.

3. Knecht H, Baumberger M, Tobon A, Steck A. Sustained remission of CIDP associated with Evans syndrome. Neurology. 2004;63 (4):730-2.

4. Marsh EA, Hirst CL, Llewelyn JG, et al. Alemtuzumab in the treatment of IVIG-dependent chronic inflammatory demyelinating polyneuropathy. J Neurol. 2010;257(6):913-9.

5. European Federation of Neurological Societies/Peripheral Nerve Society Guideline on Management of Chronic Inflammatory Demyelinating Polyradiculoneuropathy: report of a joint task force of the European Federation of Neurological Societies and the Peripheral Nerve Society. First Revision. J Peripher Nerv Syst. 2010;15:185-95.

6. Arndt PA, Leger RM, Garratty G. Serology of antibodies to secondand third-generation cephalosporins associated with immune hemolytic anemia and/or positive direct antiglobulin tests. Transfusion. 1999; 39(11-12):1239-46.

7. Stahl D, Lacroix-Desmazes S, Heudes D, Mouthon L, Kaveri SV, Kazatchkine MD. Altered control of self-reactive IgG by autologous IgM in patients with warm autoimmune hemolytic anemia. Blood. 2000; 95(1):328-35. 